\title{
Energy scaling of spin tune due to RHIC snakes
}

\author{
W. W. MacKay
}

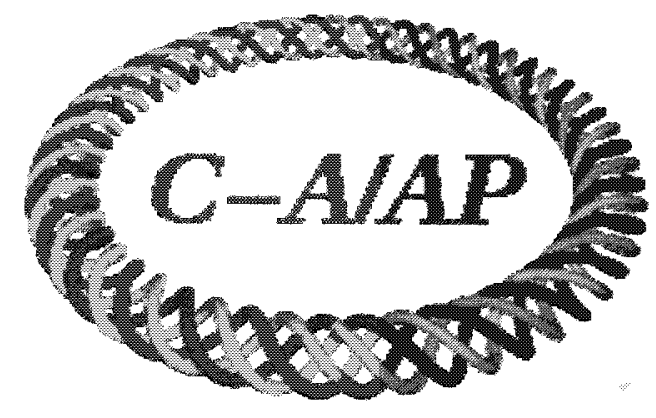

\section{Collider-Accelerator Department Brookhaven National Laboratory Upton, NY 11973}

Notice: This document has been authorized by employees of Brookhaven Science Associates, LLC under Contract No. DE-AC02-98CH10886 with the U.S. Department of Energy. The United States Government retains a nonexclusive, paid-up, irrevocable, world-wide license to publish or reproduce the published form of this document, or allow others to do so, for United States Government purposes. 


\title{
Energy scaling of spin tune due to RHIC snakes
}

\author{
W. W. MacKay
}

For a ring like RHIC with two full Siberian snakes on opposite sides of the ring, the spin tune for a flat orbit will be $1 / 2$ if the snake rotation axes are perpendicular, $\Delta \phi=\phi_{9}-\phi_{3}=\frac{\pi}{2}$. Here $\phi_{9}$ and $\phi_{3}$ are respectively the direction of the rotation axes of the 9 o'clock and 3 o'clock snakes relative to the design trajectory as shown in Fig. 1.

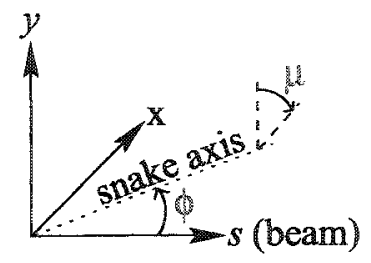

Figure 1. Definition of snake angles.

If the two snakes are slightly detuned by the same amount such that the rotation axes are no longer perpendicular, then the deviation of the closed-orbit spin tune $\nu_{0}$ from $1 / 2$ is given by

$$
\Delta \nu_{0} \simeq \frac{(\Delta \mu)^{2}}{4 \pi} \cos G \gamma \pi-\frac{2 \Delta \phi}{\pi} \simeq \frac{2 \Delta \phi}{180^{\circ}} \text { with } G \gamma \text { at a half integer }
$$

and where $\Delta \mu$ is the deviation of snake rotation angle from $180^{\circ}$. It should be noted that there is a sign ambiguity in $\Delta \nu_{0}$ since a spin tune of 0.495 is also a spin tune of 0.505 , depending on the direction taken along the stable spin axis. In order to understand the effect of energy scaling on the snake axis direction, I have integrated the trajectory and spin rotation through a model of a RHIC snake (bi9-snk7) and found the energy $(U)$ dependence of the snake axis angle $\phi_{9}$ and rotation angle $\mu$ as shown in the following table:

$\begin{array}{rcc}U[\mathrm{GeV}] & \phi_{9}[\mathrm{deg}] & \mu[\mathrm{deg}] \\ 23.812 & 134.91 & 177.57 \\ 50.000 & 135.10 & 177.39 \\ 100.000 & 135.14 & 177.35 \\ 250.000 & 135.15 & 177.34\end{array}$

$\mathrm{A} \simeq p^{-2}$ scaling of errors is typical in helical snakes. To first order, the orbit excursion drops as $p^{-1}$ and the spin precessions about transverse fields increase as $\gamma$ giving an approximate cancellation with energy, so we do not expect much change during the field ramp. The next order term which comes in is primarily proportional to $p^{-2}$; although naively one might expect a slight effect inversely proportional to the velocity since $\gamma / p \propto \frac{c}{v} \simeq 1+\frac{1}{2 \gamma^{2}}$.

The scaling of $\phi$ with energy in the previous table to a good approximation is fit by

$$
\phi(U) \simeq 135.154-\frac{137.7[\mathrm{GeV}]}{\sqrt{U^{2}-m^{2} c^{4}}}
$$

as shown in Fig. 2. A curve fit to $p^{-1}$ scaling is also shown for comparison. 


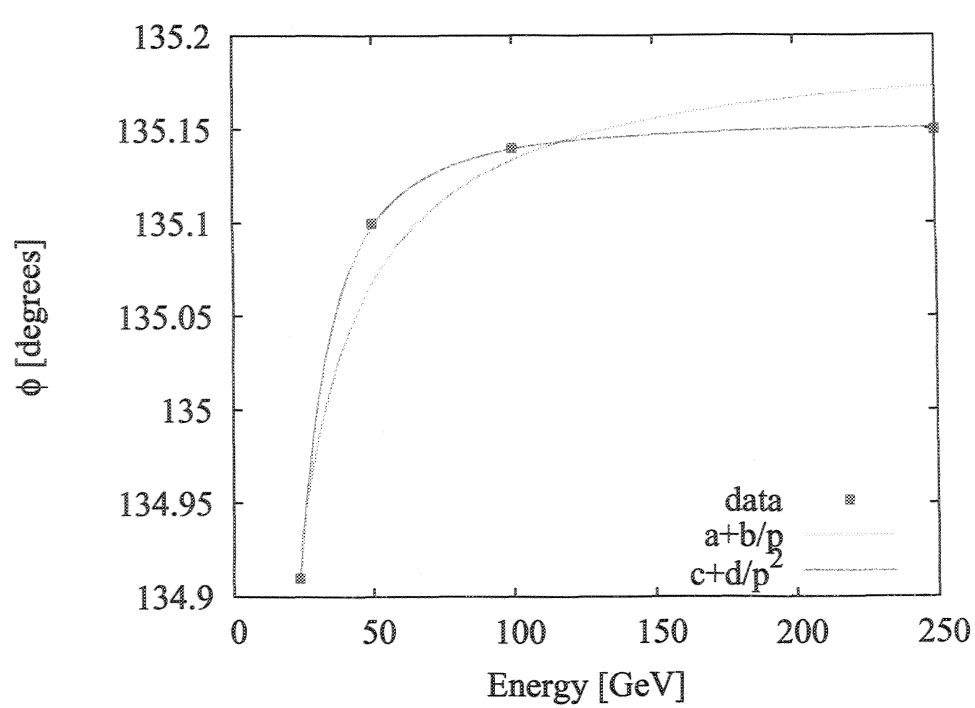

Fig. 2. Scaling of $\phi_{9}$ with energy.

In the RHIC spin design manual[1] calculations were performed with this simple snake velocity scaling that ignored the trajectory through the snake to estimate the energy dependence of the spin tune. Ignoring the trajectory produces a larger tune shift than calculating the spin precession for the actual corkscrew trajectory in the snake. Vahid Ranjbar demonstrated this when he integrated the spin and trajectory through realistic snake fields[1].

Combining Eqs. (1 and 2), the spin tune shift dependence on energy is plotted in Fig. 3.

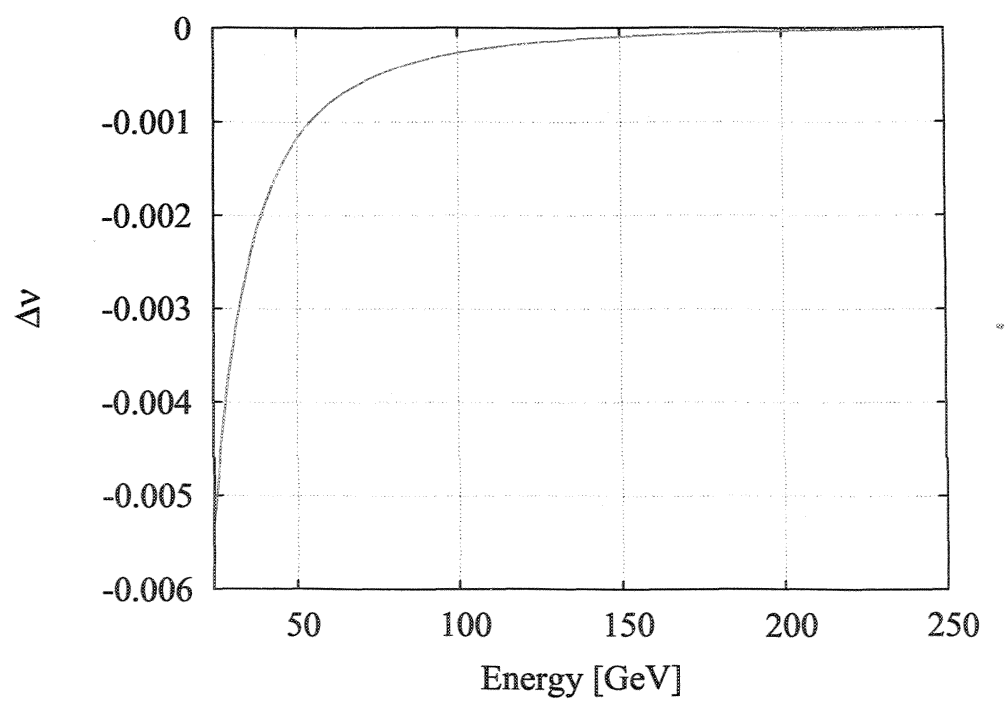

Fig. 3. Scaling of spin tune with energy.

It is perhaps worth noting that a change of $-1 \mathrm{~A}$ in the inner helices of both snakes at injection energy $(G \gamma=45.5)$ will shift the snake axis by $135.27^{\circ}-134.91^{\circ}=0.36^{\circ}$, which corresponds to a shift of $\left|\Delta \nu_{0}\right|=0.008$.

Some example spin tune variations from other effects (separation bumps, injection snake bumps, and experiment solenoids) are listed in the next table: 


\begin{tabular}{cl}
$\nu_{0}$ & \multicolumn{1}{c}{ Conditions } \\
0.50000 & ideal flat ring with two perfect snakes \\
0.50000 & +0.5 mm vertical separation bumps at all IR's \\
0.50008 & vertical separation bumps and injection snake bumps \\
0.50027 & vertical separation and snake bumps and STAR and PHENIX solenoids \\
& (STAR: 0.5 T and PHENIX 0.833 T) \\
0.50002 & STAR solenoid, snake bumps, and vertical separation bumps \\
0.50018 & PHENIX solenoid, snake bumps, and vertical separation bumps
\end{tabular}

One should note while examining this table, that the effects do not always add linearly, since there is some interference due to the noncommutativity of $\mathrm{SU}(2)$. Orbit errors at injection may yield a comparable shift of spin tune to those shown in this last table.

In the past, we seem to have had good polarization transmission, so we should not worry about trying to ramp snakes during the energy ramp to correct the low-energy shift of 0.005 . From $100 \mathrm{GeV}$ to $250 \mathrm{GeV}$, there is no appreciable shift due to snake energy scaling.

While there may be a systematic error in our understanding of the helix field as a function of current, we have used Vahid's[2] OPERA integration through his field map which was derived from the magnetic measurements of the four helices in the first snake (bi9-snk7). So far his values seem to work fairly well. Doing a snake scan across a snake resonance is not particularly helpful at injection energy, since we would have to apply a correction to shift the spin tune away from $1 / 2$. Since we do not have a good measurement of the actual spin tune and have not put in all the effects, especially orbit errors, we cannot say which direction to shift the snake currents at injection. If we guess wrong, then we might make the top energy situation worse. For example if we were to shift the spin tune away from $1 / 2$ at injection by $\Delta \nu_{0}=0.005$ when it should have been $\Delta \nu_{0}=-0.005$, then we could easily have an error of 0.01 from 100 to $250 \mathrm{GeV}$. To remove this ambiguity we need to know the direction $\left( \pm\right.$ ) taken for the stable spin vector $\hat{n}_{0}$ in addition to the spin tune $\nu_{0}$. (Perhaps the sign of $\hat{n}_{0}$ could be obtained with the new spin flipper, but simple energy scaling may in fact be swamped by orbit errors.)

If we want to calibrate the snakes it should be done at high energy ( $\geq 100 \mathrm{GeV}$ ) with an actual spin tune measurement if that turns out to be feasible. Such a spin tune measurement would best be done first without rotators at the end of the energy ramp but with the final energy ramp optics (vertical separation bumps, but no horizontal bumps) to locate the best snake setup for the ramp. Another spin-tune measurement to locate $\nu_{0}$ could be made at storage with the rotators to allow adjustments if polarization lifetime were found to be too short.

I would like to thank Vadim Ptitsyn and Christoph Montag for fruitful discussions.

Reference

1. I. Alekseev et al., "Polarized Proton Collider at RHIC", (1998). http://ww .rhichome.bnl.gov/Spin/design/

2. Vahid H. Ranjbar, Increasing the Proton Polarization in $A G S$ and $R H I C, \mathrm{Ph}$. D. Thesis, Univ. of Indiana (2002). 